\title{
Patient and Caregiver Health State Utilities in Tuberous Sclerosis Complex
}

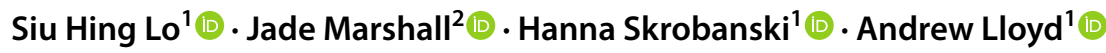

Accepted: 27 July 2021 / Published online: 15 September 2021

(c) The Author(s) 2021

\begin{abstract}
Background Tuberous sclerosis complex (TSC) is a rare multisystem disorder often associated with treatment-resistant epilepsy. Cost-effectiveness analysis for new antiseizure medications typically requires health state utilities (HSUs) that reflect the burden of a given condition.

Objective This study aimed to estimate HSUs, with a focus on valuing the impact of seizure type and seizure frequency on health-related quality of life (HRQL) for patients with TSC and their caregivers.

Methods A targeted literature review and qualitative research with healthcare professionals and caregivers informed the development of health state vignettes describing the experience of living with TSC or caring for a child with TSC. Vignettes were evaluated in interviews with the UK general population using the time trade-off (TTO) method.

Results Sixteen vignettes were developed describing patient HRQL $(n=8)$ and caregiver HRQL $(n=8)$. Two hundred interviews were conducted via online video calls due to COVID-19 pandemic restrictions. Two hundred participants evaluated the patient $(n=100)$ and caregiver $(n=100)$ health state vignettes. Estimated utility scores varied consistently according to seizure type and seizure frequency. Patient TTO utility scores ranged between -0.234 (highest seizure frequency and multiple seizure types) and 0.725 (seizure-free state). Caregiver TTO utility scores ranged from 0.221 to 0.905 .

Conclusions Findings highlight the substantial burden of living with TSC and caring for a child with TSC. Patient and caregiver burden was greater for generalised versus focal seizures. The burden was greatest for a combination of both seizure types and worsened with increasing seizure frequency.
\end{abstract}

Siu Hing Lo

siuhing.lo@acasterlloyd.com

1 Acaster Lloyd Consulting Ltd, 16 Upper Woburn Place,

Bloomsbury, London WC1H 0BS, UK

2 GW Pharma Ltd, 1 Cavendish Place, Marylebone,

London W1G 0QF, UK 


\section{Key Points for Decision Makers}

Tuberous sclerosis complex is a rare disorder, often associated with treatment-resistant epilepsy.

This study used the time trade-off (TTO) method to estimate health state utilities and found that increased seizure frequency was associated with a negative impact on health-related quality of life for both patients with tuberous sclerosis complex and their caregivers.

The TTO weights produced in this study can be used for cost-effectiveness modelling whilst adding to the literature and supporting the robustness of findings in reimbursement processes.

\section{Introduction}

Tuberous sclerosis complex (TSC) is a rare multisystem genetic disorder characterised by benign tumours in multiple organs [1]. Clinical manifestations of the disorder can vary widely between individuals with TSC and across an individual's lifetime [2]. The specific organs affected by the condition vary, but those most affected include the skin, brain, kidneys, lungs, and heart [1]. The impact of the disorder on these organs can lead to complications such as hydrocephalus, cardiac arrhythmias, and renal problems, which are a source of patient mortality [1]. TSC is also associated with neurodevelopmental and neuropsychiatric disorders such as learning difficulties, autism, behavioural difficulties, anxiety, and depression [2].

Epileptic seizures are a common manifestation of TSC, affecting more than three-quarters of individuals with this disorder [2-5]. Early TSC-associated seizures, including focal seizures and infantile spasms, generally begin during the first year of life [6]. In two-thirds of patients with TSC, the epilepsy is considered to be treatment-resistant [7]. Early onset of epilepsy as well as increased severity and higher seizure frequency are associated with neuropsychiatric disorders, such as cognitive deficits and autism spectrum disorder [8, 9]. Patients with TSC and epilepsy can experience different types of seizures, including generalised seizures and focal seizures with impaired awareness [4]. Generalised seizures are caused by abnormal electrical activity spreading throughout the brain and affecting the whole body [10]. Focal seizures with impaired awareness are caused by abnormal activity in one part of the brain and result in impaired consciousness [10]. Focal seizures can also spread to become generalised seizures [10]. Generalised seizures can be particularly hazardous, as they can cause loss of consciousness and patients to fall if unsupported [10].

The emergence of new antiseizure medications will require health technology assessment (HTA) or comparative effectiveness studies to determine relative treatment benefits for different stakeholders, including patients, caregivers, and payers. HTA reviews typically assess the cost effectiveness of treatments in terms of the gain in health measured by length of life and quality of life. Health state utilities (HSUs) quantitatively measure quality of life and reflect how different health states are viewed by society. Health gains from an effective treatment can be quantified, and effects may extend to both patients and caregivers, where there is a substantial caregiver burden associated with a condition. In TSC and childhood epileptic encephalopathies such as Lennox-Gastaut syndrome, caregivers' or parents' health-related quality of life (HRQL) has been shown to be significantly impacted as a result of caring for a child [11-13]. As one of the sources of caregiver burden [13], there could be potential improvements in HRQL if a child with refractory epilepsy receives an effective treatment. The benefit for caregivers can be included in a costeffectiveness analysis.

Different methods exist for capturing HSUs. In prevalent diseases, health status is usually measured in a patient population using EQ-5D (a self-assessed HRQL questionnaire) or similar standardised measures and converted to utilities using preferences elicited from the general population. In the present context, the use of standardised measures is more problematic. First, the EQ-5D has been criticised as a poor choice of outcome measure in seizure disorders [14, 15]. Second, TSC is a rare condition [2], which makes it practically difficult to recruit large samples of patients for HRQL estimation. Lastly, the classification of health states in TSC to evaluate antiseizure medications requires data from patients experiencing different seizure types at varying seizure frequencies. Therefore, prospective HRQL assessment with EQ-5D was considered impractical. As an alternative, a time trade-off (TTO) vignette study was conducted, whereby specific TSC health states with different types of seizures at varying frequencies were described in bespoke vignettes.

The aim of this study was to elicit preferences of HSUs, or utility weights, for patient and caregiver TSC health states, in order to estimate the gains in health associated with improved seizure control. To achieve this objective, vignette descriptions of each patient or caregiver health state were first developed. The vignettes were then assessed by the general public in the UK using TTO interview methodology. 


\section{Methods}

\subsection{Study Design}

Health state vignettes were developed to describe and present all important aspects of the experience of a child living with TSC and a caregiver caring for a child with this condition. Health state vignettes varied by number of seizures per day and type of seizures experienced (focal seizures with impaired awareness only, generalised seizures only or a combination of both seizure types).

The content of the vignettes was informed by a targeted literature review of quality-of-life publications in TSC, and qualitative research with TSC caregivers and healthcare professionals (HCPs; epilepsy specialist nurses or clinicians) with experience in management of patients with TSC. As TSC is associated with age-dependent complications [16], we focused on typical issues affecting a 13-year-old patient with TSC. The use of this patient profile ensured that the vignettes focused on patients of an age at which seizures are expected to be a significant contributing factor to the patient/ caregiver burden, based on the natural history of the condition [16]. The language in the patient vignettes was adapted to ensure adult participants were able to value the patient health states from their own perspective. For example, any references to school were swapped with the possibility of having a job at present. Caregiver vignettes described the experience of someone who is one of two primary caregivers/parents of a 13-year-old child with TSC, with some minimal references to the corresponding patient health state to contextualise the caregiver burden.

\subsection{Targeted Literature Review}

A targeted literature review was conducted to identify published data on the experience of individuals with TSC and their caregivers, with a particular focus on the impact of seizures on HRQL. The current review built on a previously published systematic review by Zöllner et al. [17] on disease burden in TSC. The original review [17] was updated by searching for publications in Medline and Embase between January and June 2020, and using expanded search terms to cover three specific areas of interest: (i) caregiver burden and impacts; (ii) patient burden and impacts; (iii) impact of seizures on patient or caregiver HRQL. Search terms are detailed in Table 1. The reference list of the previous systematic review [17] was hand searched to identify any further relevant papers.

Based on the findings of the literature review, an initial set of two draft vignettes was developed, with one vignette describing the TSC patient/caregiver experience without seizures, and another describing a state with one generalised seizure and two focal seizures with impaired awareness per day. The initial set was used as a basis for discussion with caregivers and HCPs.

\subsection{Caregiver and Healthcare Professional (HCP) Interviews}

Caregivers of patients with TSC and epilepsy were recruited via the Tuberous Sclerosis Association (TSA) in the UK [18]. HCPs with known experience in clinical management of TSC were selected by the study sponsor. Qualitative semi-structured interviews lasting approximately $1 \mathrm{~h}$ were conducted with caregivers and HCPs via online video call. The interviews provided feedback on the draft vignettes and helped capture the HRQL impact of
Table 1 Search strategy for literature review

\begin{tabular}{|c|c|}
\hline No. & Search terms \\
\hline 1 & TSC [abstract] OR "tuberous sclerosis complex" [abstract] OR tuberous sclerosis/ [abstract] \\
\hline $2^{\mathrm{a}}$ & $\begin{array}{l}\text { "burden of illness" [abstract] OR BOI [abstract] OR "health burden" [abstract] OR "health } \\
\text { care use" [abstract] OR "health care utilization" [abstract] OR "resource use" [abstract] } \\
\text { OR "resource utilization" [abstract] OR "disease-adjusted life years" [abstract] OR DALY } \\
\text { [abstract] OR "quality-adjusted life years" [abstract] OR QALY [abstract] OR "quality of } \\
\text { life" [abstract] }\end{array}$ \\
\hline $3^{\mathrm{b}}$ & $\begin{array}{l}\text { “caregiver burden" [abstract] OR "carer burden" [abstract] OR "parent burden" [abstract] } \\
\text { OR "burden on caregiver" [abstract] OR "burden on carer" [abstract] OR "burden on par- } \\
\text { ent" [abstract] OR "patient burden" [abstract] OR "seizure type" [abstract] OR "type of } \\
\text { seizure" [abstract] OR "seizure frequency" [abstract] }\end{array}$ \\
\hline 4 & 2 OR 3 \\
\hline 5 & 1 AND 4 \\
\hline
\end{tabular}

$B O I$ burden of illness, $D A L Y$ disease-adjusted life-year, $Q A L Y$ quality-adjusted life-year, TSC tuberous sclerosis complex

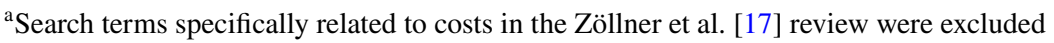

${ }^{\mathrm{b}}$ Search terms added to search strategy in the Zöllner et al. [17] review 


\section{P1: Seizure-free state}

- Your medication keeps your epilepsy under control, so you do not experience any seizures.

- You have a disease which can affect the functioning of different organs in your body, including the brain, eyes and/or skin.

- You have skin abnormalities, including on your face.

- You often feel tired and sleepy and have poor appetite.

Daily life

- You have a moderate learning disability and had specialist education and support at a mainstream school when you went to school in the past. You could have a full-time job, but your condition may limit what you can do.

- You have to regularly attend healthcare appointments for monitoring, tests and scans for your condition.

- You sometimes need to have medical interventions (e.g. surgery) due to the complications of your condition.

- You need a light level of support from caregivers to do complex tasks (e.g. organising the payment of bills) due to your learning disability.

\section{Psychological and social well-being}

- You sometimes feel depressed or anxious. You are socially withdrawn and find it difficult to make friends.

- You sometimes worry about your future due to the changeable nature of your condition, and the serious complications that could occur.

- You sometimes feel frustrated by your condition and feel self-conscious about being different to your peers.

- You sometimes have behavioural problems, which can impact on your relationships with people.

Fig. 1 Health state describing a patient with TSC in a seizure-free state. TSC tuberous sclerosis complex

living with TSC in relation to the described health states. The initial interviews with caregivers and HCPs were designed to validate and refine the content of the vignettes. The effects of increasing or decreasing the number of generalised seizures and focal seizures with impaired awareness were explored within the context of the second draft vignettes. Findings from the interviews were used to inform the definition of health states included in the final set of vignettes, to ensure that states would be sufficiently differentiated in terms of seizure frequency for each seizure type. Draft health states were iteratively revised based on notes from the caregiver and HCP interviews, ensuring that incremental changes made to the drafts were reviewed by subsequent interviewees. The final set of vignettes was reviewed by a clinician.
The caregiver vignettes were systematically compared and harmonised with the patient vignettes to ensure that they were mirrored where appropriate, and only differed in aspects where the participants' perspective (patient vs caregiver) justified variations in wording and content. The final draft vignettes are provided in Figs 1, 2, 3, 4, and S1-S12 (see electronic supplementary material [ESM]).

\subsection{Selection of Health States}

To keep the length of TTO valuation interviews manageable, vignettes were developed for each seizure type: (i) focal seizures with impaired awareness and (ii) generalised seizures, at varying daily seizure frequencies; but only one vignette with a combination of both seizure types 
P8: Between 3 and 14 generalised seizures, and between 5 and 14 focal seizures with impaired awareness

- You experience between 3 and 14 generalised seizures, and between 5 and 14 focal seizures with impaired awareness per day. You take medication for your epilepsy.

- You have a disease which can affect the functioning of different organs in your body, including the brain, eyes and/or skin.

- You experience discomfort due to your seizures.

- You have skin abnormalities, including on your face.

- You always feel tired and sleepy and have poor appetite.

Daily life

- You have a moderate learning disability and had specialist education and support at a mainstream school when you went to school in the past. You cannot have a job due to your condition.

- You have to regularly attend healthcare appointments for monitoring, tests and scans for your condition. You also have frequent regular healthcare appointments specifically for your epilepsy, as you need to try new medication to manage your seizures.

- You sometimes need to have medical interventions (e.g. surgery) due to the complications of your condition. You are also often admitted to hospital because of your seizures.

- You need an extremely high level of support from caregivers to do some daily activities (e.g. housework) and self-care (e.g. washing, toileting) due to your seizures and learning disability.

- You very often use a wheelchair due to risk of injury from your seizures.

\section{Psychological and social well-being}

- You extremely often feel depressed or anxious. You are socially withdrawn and find it difficult to make friends.

- You often worry about your future due to the changeable nature of your condition, and the serious complications that could occur.

- You extremely often feel frustrated by your condition and lack of independence and feel self-conscious about being different to your peers.

- You extremely often have behavioural problems and attention difficulties, which can impact on your relationships with people.

- You extremely often feel confused and upset due to your seizures.

Fig. 2 Health state describing a patient with TSC experiencing 3-14 generalised seizures per day, and 5-14 focal seizures with impaired awareness per day. TSC tuberous sclerosis complex

was developed (Table 2). For other possible states with a combination of seizure types, utility weights could be estimated through linear interpolation of the differences between TTO-valued states. An example of how values could be estimated using the TTO-valued states is presented in Table 3. Estimates were made assuming that disutility increases in a linear fashion with increasing seizure frequencies. Values for states with the lowest and highest number of seizures (valued using the TTO vignette method) were used as anchoring points to allow values of other states with seizure frequencies falling within this range to be estimated.

\subsection{Health State Valuation}

Members of the general public in the UK were recruited by seven interviewers based in various regions of the UK, using convenience sampling, including snowballing. The aim was 


\section{C1: Seizure-free state}

\section{Your health and daily life}

- You have no problems walking about and have no problems washing or dressing yourself as a result of needing to care for your child.

- You do not have to provide seizure-related care for your child because their medication keeps their epilepsy under control, so they do not experience any seizures.

- You need to provide your child with a light level of support to carry out some daily activities when they are not at school because of their moderate learning disability. Your daily routine is sometimes affected by the needs of your child and impacts on the rest of your family.

- You need to spend some time every day managing your child's medical needs (medication, healthcare appointments, hospital visits and stays).

- You sometimes feel tired or exhausted and lack energy to do all the things you would like to do.

- It is sometimes difficult to plan holidays or go out with friends and family because of your child's condition.

- You sometimes find it difficult to look after your own physical and mental wellbeing (e.g. exercising, maintaining a healthy diet, spending time with your partner).

- You sometimes have depressive or anxious thoughts and find your child's health problems distressing and stressful. You worry about the changeable nature of your child's condition and the serious complications that could occur. You can also feel lonely and isolated.

- You sometimes worry about your child's future and them being stigmatised due to their health problems.

Fig. 3 Health state describing a caregiver of a patient with TSC in a seizure-free state. TSC tuberous sclerosis complex

to recruit a broadly representative sample of the UK general public in terms of age, sex, and ethnicity. Participants were eligible if they were adults (aged 18 years and above). A total of 200 TTO interviews were conducted. Patient vignettes were assessed by 100 participants, and caregiver vignettes were assessed separately by 100 participants. The sample sizes are similar to many other published TTO studies [19-22]; no formal sample size calculations were performed as the study did not intend to test a specific hypothesis. In response to the COVID-19 pandemic, all interviews were conducted between August and September 2020 using online video calls (Zoom/Skype). Participants were provided with information about the study and asked to complete a consent form and a brief background questionnaire.

Participants were first asked to read the introductory text and instructions (Figs. S13-S14, see ESM), which asked them to imagine that they had the condition themselves or that they were one of two primary caregivers of a child who had the condition. Interviewers were instructed to check each participant's understanding of the exercises by asking a series of comprehension questions; the interview was terminated if they were sure that the participant did not understand. For the valuation of caregiver vignettes, participants were instructed to focus on their own HRQL and not that of their child. Participants were asked to imagine themselves in the described state and questioned whether they would trade their own years of life. They were not asked to imagine trading years of a child's life. The first exercise used a visual analogue scale (VAS) ranging from 0 (worst possible state) to 100 (full health), which measured seizure frequency/ intensity. Eight patient or caregiver vignettes and the state of 'being dead' were then presented one by one. Following the VAS exercise, participants completed the TTO exercise. Prior to the VAS and TTO exercises with the patient/ 


\section{C8: Between 3 and 14 generalised seizures, and between 5 and 14 focal seizures with impaired awareness}

\section{Your health and daily life}

- You have no problems walking about and have no problems washing or dressing yourself as a result of needing to care for your child.

- You have to provide seizure-related care for your child because they experience between 3 and 14 generalised seizures, and between 5 and 14 focal seizures with impaired awareness per day. You have to watch out for your child's seizures and monitor their length to assess whether emergency medication or assistance is required.

- You continuously need to monitor your child's activities, and assess their risk of injury, due to their seizures.

- You have to spend a very large amount of time each day reassuring your child, managing their disruptive behaviour, and/or washing and dress them, following a seizure.

- You need to provide your child with some support to carry out daily activities when they are not at school because of their moderate learning disability. Your daily routine is completely driven by the needs of your child and impacts the rest of your family.

- You have stopped working to enable you to care for your child

- You need to spend a lot of time every day managing your child's medical needs (medication, healthcare appointments, hospital visits and stays).

- You always feel tired or exhausted and lack energy to do all the things you would like to do.

- It is extremely difficult to plan holidays or go out with friends and family because of your child's condition.

- You always find it difficult to look after your own physical and mental wellbeing (e.g. exercising, maintaining a healthy diet, spending time with your partner).

- You extremely often have depressive or anxious thoughts and find your child's health problems distressing and stressful. You worry about the changeable nature of your child's condition and the serious complications that could occur. You can also feel lonely and isolated.

- You often worry about your child's future and them being stigmatised due to their health problems.

Fig. 4 Health state describing a caregiver of a patient with TSC experiencing 3-14 generalised seizures per day and 5-14 focal seizures with impaired awareness per day. TSC tuberous sclerosis complex

caregiver vignettes, as a warm-up exercise, participants were asked to rank two practice vignettes, depicting simple health states describing key domains of HRQL similar to those covered in the EQ-5D.

Vignettes were either printed or presented to the participants on a screen in a quasi-random order. For those who viewed the vignettes on the screen, the online video interview was conducted on a second screen so that the interviewer and participant were always visible to one another. This enabled the interviewers to show the paper VAS and wooden TTO board alongside themselves on the screen. The online interview was designed to mimic a standard face-toface interview as closely as possible. All interviews were conducted by trained TTO interviewers.

The TTO interview is a standardised method for valuing health states and was used to generate utility weights for each vignette [23, 24]. During the interview, the participants were asked to choose their preference between the prospect of living for 10 years in the health state or ' $\mathrm{X}$ ' years in full health. The time in full health was systematically altered 
Table 2 Overview of health states described in TTO vignettes

\begin{tabular}{lll}
\hline Health state & \multicolumn{2}{l}{ Seizure type and frequency } \\
\cline { 2 - 3 } & $\begin{array}{l}\text { Generalised seizures, } \\
\text { no. per day }\end{array}$ & $\begin{array}{l}\text { Focal seizures with } \\
\text { impaired awareness, no. } \\
\text { per day }\end{array}$ \\
\hline HS1 & 0 & 0 \\
HS2 & 0 & $1-2$ \\
HS3 & 0 & $3-4$ \\
HS4 & 0 & $5-14$ \\
HS5 & 1 & 0 \\
HS6 & 2 & 0 \\
HS7 & $3-14$ & 0 \\
HS8 & $3-14$ & $5-14$ \\
\hline
\end{tabular}

$H S$ health state, TTO time trade-off

until the participant was indifferent between the choices. This duration of full health indicated the value or utility of the health state. To minimise possible bias, the ping-pong method was used, and the amount of time in full health was alternated between high and low values, decreasing by 6-month intervals. If the participant indicated that 'being dead' was preferable to any time living in a health state, then this indicated that they considered the state as worse than 'being dead'. At this point, the interviewer switched to a lead-time TTO exercise, which began with asking participants whether they would prefer to live for 10 years in full health followed by 10 years in a health state, or to live for 10 years in full health only [25]. The time in full health remained constant, but the number of years in a health state was systematically altered until the participant was indifferent between the choices. This lead-time procedure allowed the participant to trade more years of life and determined how much worse than being dead they considered the health state to be.

The VAS ratings for each vignette were rescaled such that the value for the state of 'being dead' was fixed at 0 , and all other values varied between 100 and the worse health state. The following formula was used to rescale the data:
$V^{\prime}=\left(\left(V-V \_\right.\right.$Dead $) /\left(100-V \_\right.$Dead $\left.)\right) \times 100$,

where $V^{\prime}$ is the rescaled VAS value, $V$ is the original VAS value and $V \_$Dead is the value given to the state of 'being dead'. After rescaling, the VAS data were summarised descriptively. The TTO data were scored according to the point of indifference ( $1=$ full health; $0=$ dead), and were summarised descriptively.

\section{Results}

\subsection{Literature Review}

The database search of publications in the last year yielded a total of 461 hits. All records were screened, and additional studies were identified from reference lists of the previously published review [17]. Sixty-two full-text articles were assessed for relevance, of which 35 were included in the review. Data were extracted on the overall HRQL impact of TSC, as well as the specific impact of seizures on patients and their caregivers. Literature review findings that informed the content of the vignettes are summarised below.

\subsubsection{Patient Health-Related Quality of Life (HRQL)}

Overall, HRQL is significantly impacted in patients with TSC [16, 26-30]. Given that many organs can be affected by the disease, patients with TSC experience frequent hospitalisations, medical visits, and prescription drug use [16, 31-38]. Patients with TSC have reported experiencing pain and discomfort [26, 39]. Problems during sleep, including breathing difficulties, unusual sleeping episodes such as nightmares, and sleep walking and talking are common in TSC [13]. TSC may also be associated with hyperactivity, attention problems, anxiety, and depression [39-41]. Patients with TSC commonly face difficulty in socialising and forming relationships, resulting in feelings of loneliness, isolation, and social withdrawal [40, 42]. Facial disfigurement associated with facial angiofibromas may add to the perceived stigma of the disease [43, 44].

Table 3 Estimation of utility values using TTO-valued health states

\begin{tabular}{llllll}
\hline & & \multicolumn{4}{l}{ No. of focal seizures with impaired awareness per day } \\
\cline { 3 - 6 } & & 0 & $1-2$ & $3-4$ & $5-14$ \\
\hline No. of generalised seizures per day & 0 & HS1 & HS2 & HS3 & HS4 \\
& 1 & HS5 & HS5-(Diff (HS7, HS8)/2) & HS5-(Diff (HS7, HS8) \\
& 2 & HS6 & HS6-(Diff (HS7, HS8)/2) & HS6-(Diff (HS7, HS8) \\
& $3-14$ & HS7 & Mean (HS7, HS8) & HS8 \\
\hline
\end{tabular}

Italic text indicates estimation of utility values for states not valued in the time trade-off study

Diff difference between health states, $H S$ health state, $T T O$ time trade-off 
Seizures in TSC have a significant negative impact on patient HRQL $[26,45]$. Patients who have TSC with epilepsy have been found to have a significantly lower HRQL than those without epilepsy [45]. Burdensome after-effects of seizures included disrupted learning ability, fatigue, hemiplegia, and inability to communicate, eat, sleep, or relax for up to $24 \mathrm{~h}$ after an episode [46]. Patients reported frustration with their lack of independence due to their risk of seizures [46]. Another study has found that adult patients with TSC reported a loss of work productivity because of their condition [47].

HRQL has additionally been shown to worsen with increasing seizure frequency and severity [28, 29, 48]. Studies exploring the effectiveness of treatments for seizures in TSC have found seizure freedom and reduction in seizure frequency to be related to improvements in patient HRQL [49-54]. Recent qualitative findings also suggest that a reduction in seizure frequency and severity has a positive impact on HRQL, including improved attention, memory, mood, and energy [46].

\subsubsection{Caregiver HRQL}

Overall, caregivers of patients with TSC have a significantly lower HRQL and more depressive symptoms than healthy adult populations [13]. Caregivers have reported fearing their unknown future, and the disease becoming increasingly unsustainable over time $[42,55]$. Caregivers have additionally voiced their anxiety over having to navigate through medical emergencies, the appearance of new symptoms, repeated surgeries, and severe side effects of therapies [46, 55]. Patients' facial angiofibromas may intensify caregivers' worries and worsen perceived stigma of the disease $[43,44]$. The neurodevelopmental and neuropsychiatric manifestations of TSC can also be a major stressor for caregivers $[41,56]$. One study found that although $65.9 \%$ of carers of children with TSC were employed, more than half perceived the disease to have impacted on their career [36]. Employed caregivers of patients with TSC reported missing work and impaired productivity due to caregiving [47]. TSC can greatly impact on the HRQL of the whole family, with caregivers reporting changes to the family's daily routines to centre around the patient's needs [55].

Seizures in TSC have a negative impact on caregiver burden $[53,54]$. Following treatment, reduced seizure frequency in children with TSC is significantly associated with increased parent satisfaction [54]. Another study found parent-reported reduced seizure frequency to be associated with improved family functioning and reduced time in managing the epilepsy of patients with TSC [53]. When seizure frequency and severity were reduced in patients with TSC, their parents reported more time for respite and family activities and less time needed to monitor the patient [46].

\subsection{Health State Development Interviews}

Four caregivers of patients with TSC and epilepsy, and three HCPs with expertise in TSC were interviewed. The four caregivers were parents of children with the condition aged between 2 and 16 years. Two HCPs were epilepsy specialist nurses, who reviewed the initial set of draft vignettes. The third HCP, who reviewed the final set of eight draft vignettes, was a clinician and professor of paediatric neurology, and an expert in the treatment of epileptic syndromes.

Key revisions made to the introductory text in response to caregiver and HCP feedback are summarised in Table 4 . The main changes made to the patient and caregiver vignettes are summarised in Tables 5 and 6. During the interviews, caregivers described how their time spent monitoring their child's seizures, assessing their child's risk of injury, and responding to the after-effects of seizures (e.g. providing reassurance and managing disruptive behaviour) affected their daily routine and ability to make plans. Caregivers also described how either they or their partners had to give up work or reduce their working hours to be able to look after their child. Caregivers agreed that the impact of their child's condition on their daily life and emotional well-being (e.g. depressive or anxious thoughts) would be increased if their child experienced a greater number of daily seizures. Simultaneously, they described how their child's seizures were only one of many impacts of TSC.

Similarly, HCPs described how the child's dependence on their caregiver would increase with greater daily seizure frequency, due to their increased risk of injury (e.g. assistance with cooking to prevent a burn due to a seizure). Therefore, the HCPs agreed that children experiencing a greater number of daily seizures would feel more frustrated by their condition and lack of independence, and have more difficulties making friends and socialising. HCPs also mentioned that children with more seizures would have a higher number of hospital admissions and medical appointments. Finally, HCPs described the after-effects of different types of seizures. Focal seizures with impaired awareness were described as affecting the child's behaviour and ability to learn and retain information; generalised seizures were described as resulting in extreme exhaustion, weakness of the body, and the need to sleep.

\subsection{Health State Valuation}

Demographic characteristics of the participants who took part in the TTO valuation interviews are presented in Table 7, along with data for age, sex, and ethnicity from the most recent UK census 2011 data [57]. The UK sample characteristics were broadly representative of the UK census data in terms of age, sex, and ethnicity. 


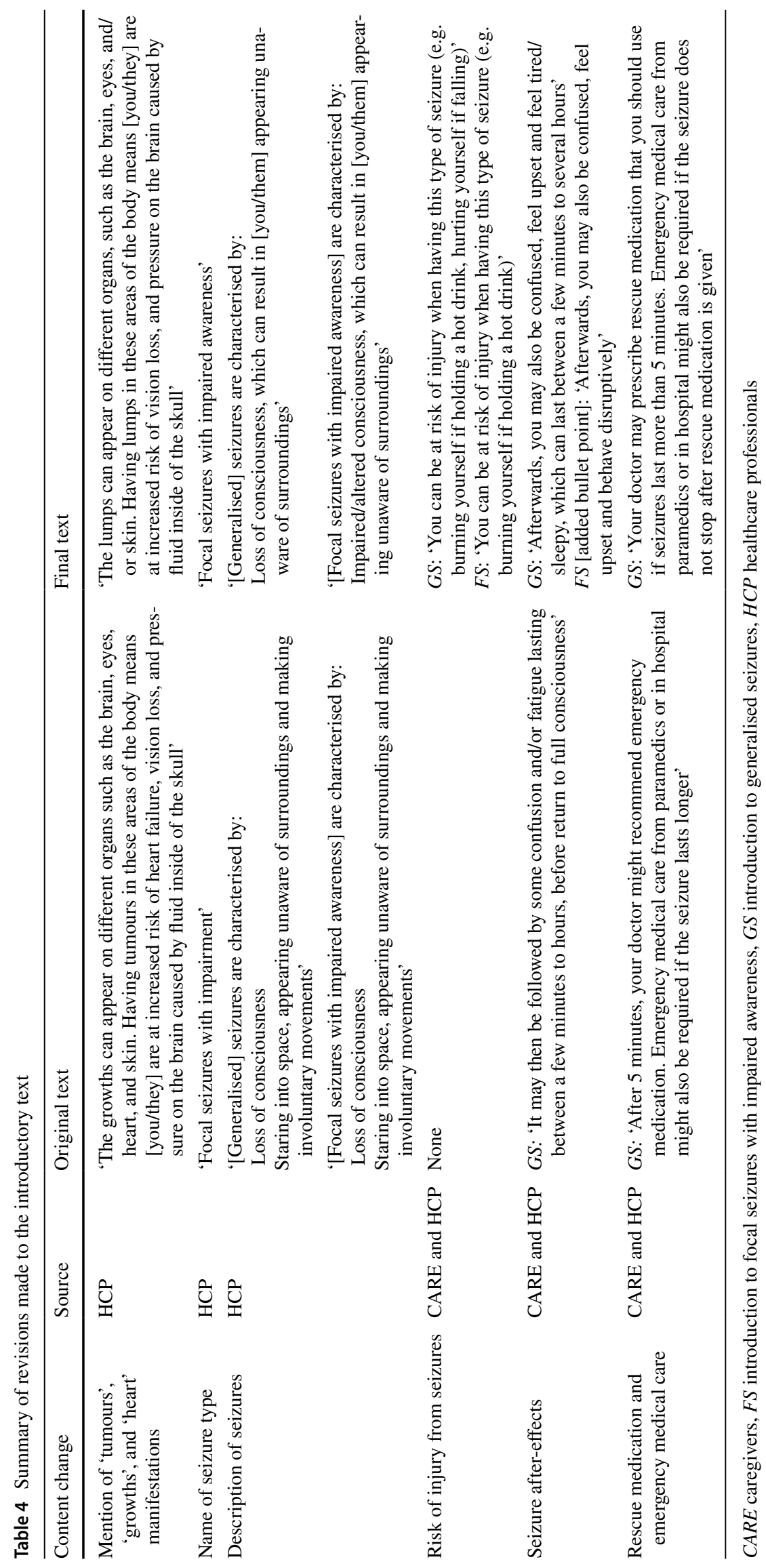




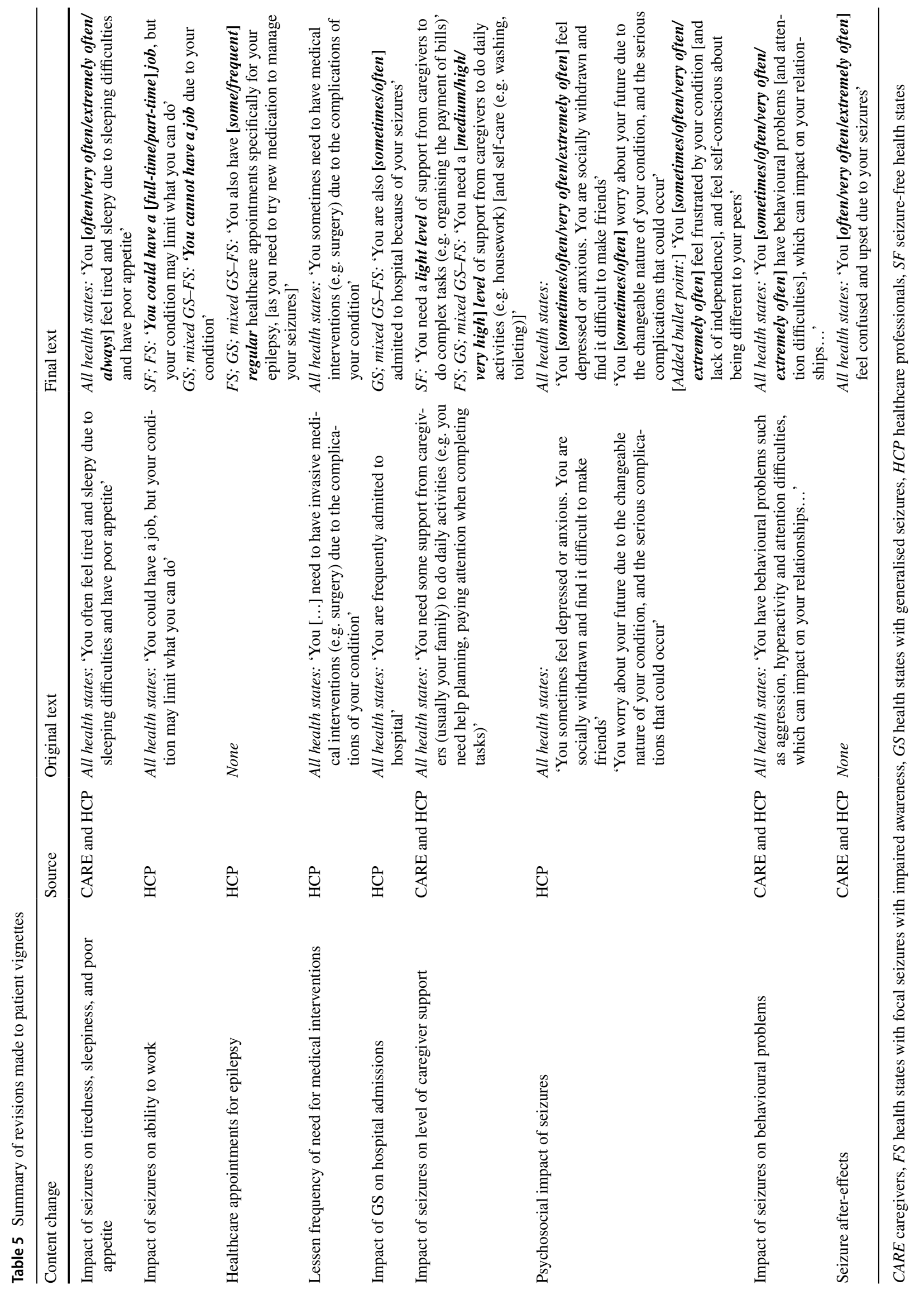




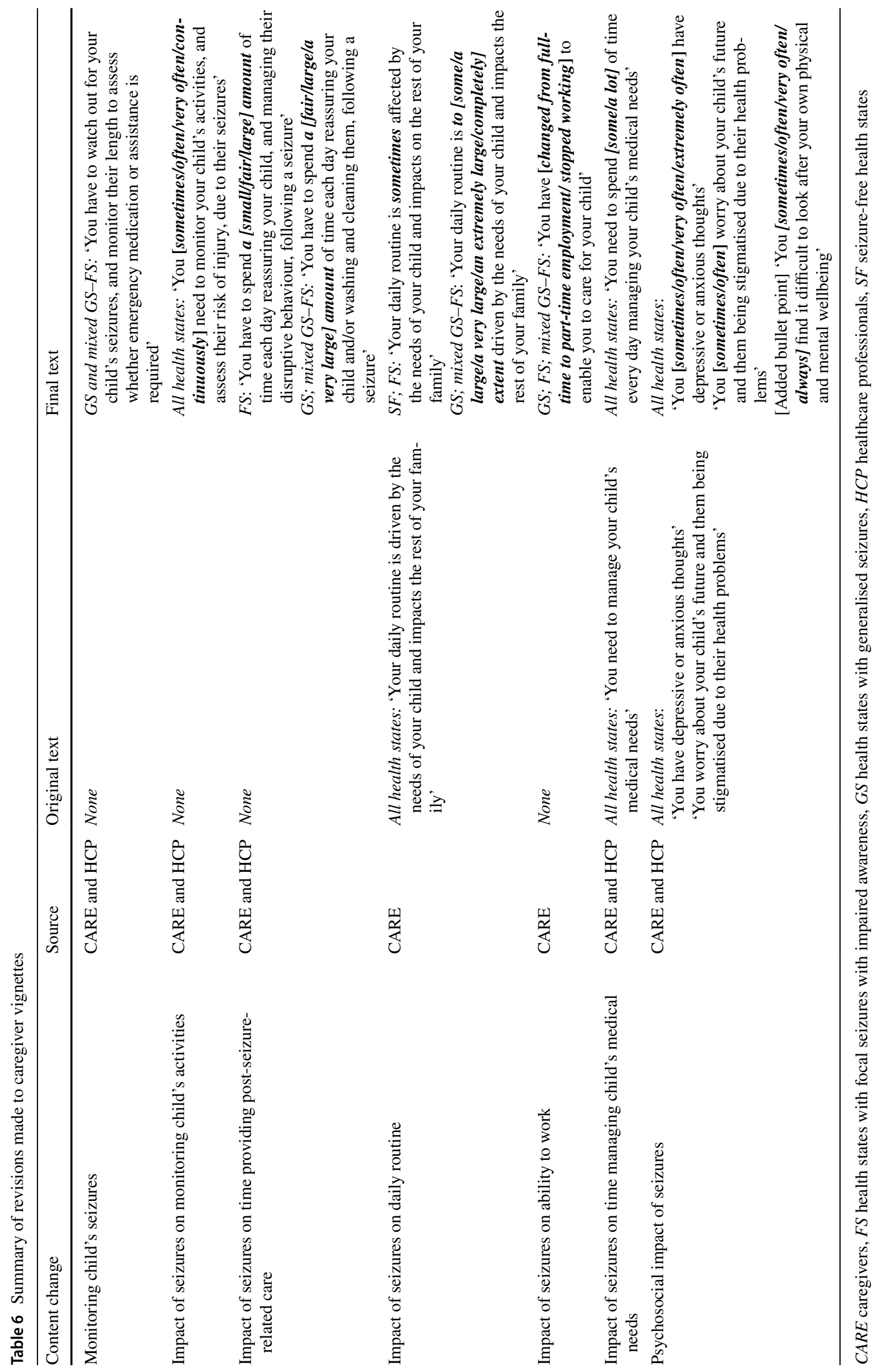


Table 7 Demographic characteristics of TTO interview participants and UK general population

\begin{tabular}{llll}
\hline Characteristic & $\begin{array}{l}\text { Participants_caregiver } \\
\text { vignettes }(n=100)\end{array}$ & $\begin{array}{l}\text { Participants-patient } \\
\text { vignettes }(n=100)\end{array}$ & UK population ${ }^{\mathrm{a}}$ \\
\hline $\begin{array}{l}\text { Age, mean (SD) } \\
\text { Sex, } n(\%)\end{array}$ & $43.58(17.18)$ & $39.49(15.62)$ & $39.4^{\mathrm{b}}$ \\
Male & $51(51)$ & $46(46)$ & $49 \%$ \\
Female & $49(49)$ & $54(54)$ & $51 \%$ \\
Ethnicity, $n(\%)$ & & & \\
White & $77(77)$ & $74(74)$ & $86 \%$ \\
Asian & $16(16)$ & $10(10)$ & $3 \%$ \\
Black & $2(2)$ & $9(9)$ & $2 \%$ \\
Mixed & $4(4)$ & $7(7)$ & \\
Other & $1(1)$ & $0(0)$ & \\
Occupation, $n(\%)$ & & & \\
Employed full-time & $40(40)$ & $55(55)$ & \\
Employed part-time & $25(25)$ & $16(16)$ & \\
Retired & $19(19)$ & $11(11)$ & \\
Student & $5(5)$ & $7(7)$ & \\
Unemployed & $7(7)$ & $7(7)$ & \\
Stay at home/carer & $4(4)$ & $1(1)$ & \\
Long-term sick leave & $0(0)$ & $2(2)$ & \\
Other & $0(0)$ & & \\
\hline
\end{tabular}

$S D$ standard deviation, TTO time trade-off

${ }^{a}$ Figures based on data from the 2011 United Kingdom national census[57]

${ }^{\mathrm{b}}$ Median

Table 8 VAS ratings and TTO weights for health-state vignettes

\begin{tabular}{|c|c|c|c|c|c|c|c|c|}
\hline \multirow[t]{2}{*}{ Health state } & \multirow{2}{*}{$\begin{array}{l}\text { No. of general- } \\
\text { ised seizures per } \\
\text { day }\end{array}$} & \multirow{2}{*}{$\begin{array}{l}\text { No. of focal seizures with } \\
\text { impaired awareness per day }\end{array}$} & \multicolumn{3}{|l|}{ VAS ratings } & \multicolumn{3}{|l|}{ TTO weights } \\
\hline & & & Mean (SD) & SE & $95 \% \mathrm{CI}$ & Mean (SD) & SE & $95 \% \mathrm{CI}$ \\
\hline \multicolumn{9}{|c|}{ Patient health states $(n=100)$} \\
\hline $\mathrm{P} 1$ & 0 & 0 & $61.50(19.65)$ & 1.96 & 57.60 to 65.40 & $0.725(0.253)$ & 0.025 & 0.674 to 0.775 \\
\hline $\mathrm{P} 2$ & 0 & $1-2$ & 45.37 (19.93) & 1.99 & 41.42 to 49.33 & $0.504(0.371)$ & 0.037 & 0.431 to 0.578 \\
\hline $\mathrm{P} 3$ & 0 & $3-4$ & $36.44(21.53)$ & 2.15 & 32.17 to 40.71 & $0.282(0.535)$ & 0.053 & 0.176 to 0.388 \\
\hline $\mathrm{P} 4$ & 0 & $5-14$ & $26.13(20.45)$ & 2.04 & 22.07 to 30.19 & $0.074(0.551)$ & 0.055 & -0.036 to 0.183 \\
\hline $\mathrm{P} 5$ & 1 & 0 & $32.19(21.66)$ & 2.17 & 27.89 to 36.49 & $0.183(0.569)$ & 0.057 & 0.070 to 0.296 \\
\hline P6 & 2 & 0 & $26.47(22.68)$ & 2.27 & 21.97 to 30.97 & $0.089(0.538)$ & 0.054 & -0.018 to 0.196 \\
\hline $\mathrm{P} 7$ & $3-14$ & 0 & $15.70(23.87)$ & 2.39 & 10.96 to 20.44 & $-0.113(0.592)$ & 0.059 & -0.231 to 0.004 \\
\hline P8 & $3-14$ & $5-14$ & $9.93(24.22)$ & 2.42 & 5.13 to 14.74 & $-0.234(0.560)$ & 0.056 & -0.345 to -0.122 \\
\hline \multicolumn{9}{|c|}{ Caregiver health states $(n=100)$} \\
\hline $\mathrm{C} 1$ & 0 & 0 & $74.16(15.00)$ & 1.50 & 71.18 to 77.14 & $0.905(0.083)$ & 0.008 & 0.890 to 0.921 \\
\hline $\mathrm{C} 2$ & 0 & $1-2$ & $58.90(17.80)$ & 1.78 & 55.37 to 62.43 & $0.791(0.171)$ & 0.017 & 0.757 to 0.825 \\
\hline $\mathrm{C} 3$ & 0 & $3-4$ & $50.21(18.44)$ & 1.84 & 46.55 to 53.87 & $0.638(0.365)$ & 0.037 & 0.565 to 0.710 \\
\hline $\mathrm{C} 4$ & 0 & $5-14$ & $34.41(19.92)$ & 1.99 & 30.46 to 38.36 & $0.431(0.494)$ & 0.049 & 0.332 to 0.529 \\
\hline $\mathrm{C} 5$ & 1 & 0 & $41.45(19.50)$ & 1.95 & 37.59 to 45.32 & $0.546(0.395)$ & 0.039 & 0.467 to 0.624 \\
\hline C6 & 2 & 0 & 36.94 (19.89) & 1.99 & 32.99 to 40.89 & $0.476(0.453)$ & 0.045 & 0.386 to 0.566 \\
\hline $\mathrm{C} 7$ & $3-14$ & 0 & $27.40(17.76)$ & 1.78 & 23.87 to 30.92 & $0.319(0.481)$ & 0.048 & 0.223 to 0.414 \\
\hline $\mathrm{C} 8$ & $3-14$ & $5-14$ & $20.67(18.77)$ & 1.88 & 16.94 to 24.39 & $0.221(0.530)$ & 0.053 & 0.115 to 0.326 \\
\hline
\end{tabular}

$C$ caregiver, $C I$ confidence interval, $P$ patient, $S D$ standard deviation, $S E$ standard error, TTO time trade-off, VAS visual analogue scale 


\subsubsection{TSC Patient and Caregiver Vignettes: VAS and TTO Ratings}

TSC patient and caregiver VAS ratings and TTO utility scores are presented in Table 8. Distribution of the TTO scores is shown in Figs. S15-S16 (see ESM). The patient TTO utility scores ranged from -0.234 (P8, presence of a combination of generalised seizures and focal seizures with impaired awareness and the highest seizure frequency) to 0.725 (P1, the seizure-free state). The caregiver TTO utility scores ranged from 0.221 (C8, presence of a combination of generalised seizures and focal seizures with impaired awareness and the highest seizure frequency) to 0.905 (C1, the seizure-free state). VAS ratings were consistent with TTO weights: states with higher frequencies of a given seizure type were valued lower than states with lower frequencies of the same seizure type, and states with generalised seizures were valued lower than states with focal seizures with impaired awareness.

Consistency of valuations was assessed by comparing utility scores between seizure-free states and states with seizures. Utility scores of individual participants were considered consistent if the seizure-free state was valued higher than all states with seizures. For the patient valuations, 94\% of VAS and $94 \%$ of TTO valuations were consistent. For the caregiver valuations, $95 \%$ of VAS and $96 \%$ of TTO valuations were consistent.

\section{Discussion}

This manuscript reports the findings of a vignette-based utility survey, which was designed to estimate the impact of different seizure types and number of seizures per day on the quality of life experienced by patients with TSC and their caregivers. The vignettes described TSC health states with focal seizures with impaired awareness only, generalised seizures only, or a combination of both seizure types. Health state vignettes were developed based on a targeted literature review, and interviews with TSC caregivers and experienced HCPs. This approach is in line with recent best practice guidelines for developing vignette studies, as published in the National Institute for Health and Care Excellence (NICE) Task and Finish Group report [58] and based on Decision Support Unit (DSU) recommendations [59]. The vignette development interviews aimed to ensure that the vignettes were easy to read, included information on all the generic dimensions of HRQL, and that the states were sufficiently differentiated without exaggerating differences. However, the vignettes were not formally tested in a separate pilot study among the general population prior to the main valuation study.

The patient vignettes included descriptions of the seizures experienced, other TSC-related clinical manifestations, comorbidities, and impacts on all major HRQL domains. This ensured that participants were given information about the typical experience of living with the condition. The caregiver vignettes described the caregiver's health and the impact of caring for a child with TSC on their daily life, with minimal references to the patient where appropriate to contextualise the described caregiver impact.

The health state vignettes were evaluated by members of the general public in the UK using VAS and TTO ratings. The values declined in line with increasing daily seizure frequency for both focal seizures with impaired awareness and generalised seizures; values were lowest for a state describing the presence of both seizure types at the highest daily seizure frequency. This highlights the extent of the perceived burden of seizures for patients with TSC and their caregivers. Accordingly, these findings show the value of an effective treatment for seizures in this complex condition, which is often associated with treatment-resistant epilepsy.

The findings of the present study are consistent with the substantial impact of epileptic seizures on quality of life in TSC, as reported by studies using patient-reported and caregiver proxy-reported generic HRQL measures to derive utility values $[45,48]$. The range of utility values for states with the highest seizure frequency in this study was comparable to the median utility scores found by Vergeer et al. [45] in patients with TSC-associated epilepsy who had refractory epilepsy at follow-up ( -0.234 to 0.074 vs -0.11$)$. Our values for the seizure-free state are also comparable to the values of Vergeer et al. [45] for patients with no seizures at followup $(0.725$ vs 0.71$)$. Furthermore, our study found that generalised seizures were associated with lower utility values than focal seizures with impaired awareness ( 1 generalised seizure per day: 0.183 vs $1-2$ focal seizures per day: 0.504), similar to Tritton et al. (0.194 [generalised seizures] vs 0.454 [complex partial seizures]) [48].

While the estimated utility values from the current study are consistent with findings from previous studies, a degree of uncertainty around the values should be acknowledged. This is evident in the $95 \%$ confidence intervals that overlap across a number of severe health states, as a result of the relatively large variability in valuations given by participants and the study sample size. While the vignettes may have good content validity, it is often difficult to convey fully the impact on HRQL in vignette descriptions. Imagining how seizures affect HRQL may be difficult for people with limited or no experience of epilepsy. Severe disease states may be more challenging for members of the general public to value because it is difficult to imagine such a state. Furthermore, there is wide variation in the clinical manifestations experienced by patients with TSC, and these can differ across their lifespan [17]. The health state vignettes were therefore designed to depict a 'typical' experience for a patient or caregiver by focusing on the clinical 
manifestations and impacts that have been most commonly found to affect patients aged around 13 years (i.e. impacts and comorbidities associated with tumours affecting the brain, eyes, and skin). Vignettes represented a simplification of individuals' experiences with the disorder, which can vary greatly across patients with TSC and their caregivers [46]. Findings from the study should therefore be interpreted in light of this limitation of the TTO vignette valuation method.

One should note that all TTO interviews were conducted via online video calls during the COVID-19 pandemic. We have no evidence that the switch to online interviews affected the data quality. To minimise the impact of this change in data collection methods, the online interviews preserved as many features of a standard face-to-face TTO interviewing experience as possible. The study results demonstrated good internal validity, consistent with differences in type of seizures and seizure frequency. Although the use of online video for TTO interviews has not yet been formally validated, a recent TTO study suggests that video interviews yield similar results to face-to-face interviews and might be a feasible alternative [60].

\section{Conclusions}

Health state vignettes were developed to describe TSC with varying seizure types and seizure frequency. Matched states were developed to describe the life of a caregiver looking after a patient with TSC. The quality of life of these states was rated by the UK general public in a TTO valuation task. This produced logically consistent TTO weights, which can be used in cost-effectiveness modelling. The study findings highlight the substantial burden of living with TSC and caring for a child with TSC. Patient and caregiver burden were greater for generalised than focal seizures. The burden was greatest for a combination of both seizure types and worsened with increasing seizure frequency. The TTO vignette methodology has limitations but can be useful for estimating utility weights in rare diseases, when observational research is challenging.

Supplementary Information The online version contains supplementary material available at https://doi.org/10.1007/s41669-021-00296-1.

Acknowledgements The authors would like to thank the participants who took part in this study. The authors would like to thank the clinical experts-Christine Bennett, Melesina Goodwin and Professor Rima Nabbout-for providing support with vignette content development. The authors would like to thank the Tuberous Sclerosis Association for helping with the recruitment of caregivers. Editorial support was provided to authors by Azka Ashraf of Helios Medical Communications, Macclesfield, UK and funded by GW Research Ltd, Cambridge, UK.

\section{Declarations}

Funding This study was funded by GW Pharmaceuticals, Cambridge, UK.

Conflicts of interest SHL, AL and HS are employees of Acaster Lloyd Consulting Ltd. JM is an employee of GW Pharma Ltd.

Availability of data and material The sponsor is adhering to current US and EU requirements so will not make individual deidentified participant data available; however, the protocol and statistical analysis plan will be made available upon request to the corresponding author.

Code availability Not applicable.

Ethics approval This study was reviewed and received exempt status determination by the Western Institutional Review Board on 7 August 2020, prior to participant recruitment.

Consent to participate Informed consent was obtained from all individual participants.

Consent for publication Not applicable.

Author contributions All authors met the ICMJE authorship criteria and had full access to relevant data. Neither honoraria nor payments were made for authorship. All authors were involved in the conception and design of the study. SHL, AL, and HS conducted the study and analysed the data. All authors revised the paper critically for intellectual content before providing final approval of the version to be published. All authors agree to be accountable for all aspects of the work.

Open Access This article is licensed under a Creative Commons Attribution-NonCommercial 4.0 International License, which permits any non-commercial use, sharing, adaptation, distribution and reproduction in any medium or format, as long as you give appropriate credit to the original author(s) and the source, provide a link to the Creative Commons licence, and indicate if changes were made. The images or other third party material in this article are included in the article's Creative Commons licence, unless indicated otherwise in a credit line to the material. If material is not included in the article's Creative Commons licence and your intended use is not permitted by statutory regulation or exceeds the permitted use, you will need to obtain permission directly from the copyright holder. To view a copy of this licence, visit http://creativecommons.org/licenses/by-nc/4.0/.

\section{References}

1. Northrup H, Krueger DA, International Tuberous Sclerosis Complex Consensus Group. Tuberous sclerosis complex diagnostic criteria update: recommendations of the 2012 international tuberous sclerosis complex consensus conference. Pediatr Neurol. 2013;49:243-54.

2. Kingswood JC, d'Augères GB, Belousova E, Ferreira JC, Carter $\mathrm{T}$, Castellana R, et al. TuberOus SClerosis registry to increase disease Awareness (TOSCA) - baseline data on 2093 patients. Orphanet J Rare Dis. 2017;12:2.

3. Nabbout R, Belousova E, Benedik MP, Carter T, Cottin V, Curatolo $\mathrm{P}$, et al. Epilepsy in tuberous sclerosis complex: findings from the TOSCA study. Epilepsia Open. 2019;4:73-84. 
4. Jeong A, Wong M. Systemic disease manifestations associated with epilepsy in tuberous sclerosis complex. Epilepsia. 2016;57:1443-9.

5. Song J, Swallow E, Said Q, Peeples M, Meiselbach M, Signorovitch $\mathrm{J}$, et al. Epilepsy treatment patterns among patients with tuberous sclerosis complex. J Neurol Sci. 2018;391:104-8.

6. Curatolo P, Jóźwiak S, Nabbout R. Management of epilepsy associated with tuberous sclerosis complex (TSC): clinical recommendations. Eur J Paediatr Neurol. 2012;16:582-6.

7. Chu-Shore CJ, Major P, Camposano S, Muzykewicz D, Thiele EA. The natural history of epilepsy in tuberous sclerosis complex. Epilepsia. 2010;51:1236-41.

8. Tye C, Mcewen FS, Liang H, Underwood L, Woodhouse E, Barker $\mathrm{ED}$, et al. Long-term cognitive outcomes in tuberous sclerosis complex. Dev Med Child Neurol. 2020;62:322-9.

9. de Vries PJ, Wilde L, de Vries MC, Moavero R, Pearson DA, Curatolo P. A clinical update on tuberous sclerosis complexassociated neuropsychiatric disorders (TAND). Am J Med Genet C Semin Med Genet. 2018;178:309-20.

10. Stafstrom CE, Carmant L. Seizures and epilepsy: an overview for neuroscientists. Cold Spring Harb Perspect Med. 2015;5:a022426.

11. Gallop K, Wild D, Nixon A, Verdian L, Cramer JA. Impact of Lennox-Gastaut syndrome (LGS) on health-related quality of life (HRQL) of patients and caregivers: literature review. Seizure. 2009;18:554-8.

12. Gallop K, Wild D, Verdian L, Kerr M, Jacoby A, Baker G, et al. Lennox-Gastaut syndrome (LGS): development of conceptual models of health-related quality of life (HRQL) for caregivers and children. Seizure. 2010;19:23-30.

13. Rentz AM, Skalicky AM, Pashos CL, Liu Z, Magestro M, Pelletier CL, et al. Caring for children with tuberous sclerosis complex: what is the physical and mental health impact on caregivers? J Child Neurol. 2015;30:1574-81.

14. Wijnen BF, Mosweu I, Majoie MH, Ridsdale L, de Kinderen RJ, Evers SM, et al. A comparison of the responsiveness of EQ-5D-5L and the QOLIE-31P and mapping of QOLIE-31P to EQ-5D-5L in epilepsy. Eur J Health Econ. 2018;19:861-70.

15. Mukuria C, Young T, Keetharuth A, Borghs S, Brazier J. Sensitivity and responsiveness of the EQ-5D-3L in patients with uncontrolled focal seizures: an analysis of Phase III trials of adjunctive brivaracetam. Qual Life Res. 2017;26:749-59.

16. Rentz AM, Skalicky AM, Liu Z, Wheless JW, Dunn DW, Frost MD, et al. Tuberous sclerosis complex: a survey of health care resource use and health burden. Pediatr Neurol. 2015;52:435-41.

17. Zöllner JP, Franz DN, Hertzberg C, Nabbout R, Rosenow F, Sauter $\mathrm{M}$, et al. A systematic review on the burden of illness in individuals with tuberous sclerosis complex (TSC). Orphanet J Rare Dis. 2020;15:23.

18. The Tuberous Sclerosis Association (TSA) [Internet]. https://tuber ous-sclerosis.org/author/nsqj26bxbz/. Accessed 11 Dec 2020.

19. Hall F, de Freitas HM, Kerr C, Ito T, Nafees B, Lloyd AJ, et al. Estimating utilities/disutilities for high-risk metastatic hormonesensitive prostate cancer (mHSPC) and treatment-related adverse events. Qual Life Res. 2019;28:1191-9.

20. Kosmas CE, Shingler SL, Samanta K, Wiesner C, Moss PAH, Becker U, et al. Health state utilities for chronic lymphocytic leukemia: importance of prolonging progression-free survival. Leuk Lymphoma. 2015;56:1320-6.

21. Nafees B, Lloyd AJ, Dewilde S, Rajan N, Lorenzo M. Health state utilities in non-small cell lung cancer: an international study. Asia Pac J Clin Oncol. 2017;13:e195-203.

22. Fordham BA, Kerr C, de Freitas HM, Lloyd AJ, Johnston K, Pelletier CL, et al. Health state utility valuation in radioactive iodinerefractory differentiated thyroid cancer. Patient Prefer Adherence. 2015;9:1561-72.
23. Drummond $\mathrm{M}, \mathrm{McGuire} \mathrm{A}$, editors. Economic evaluation in health care: merging theory with practice. Illustrated. Oxford: OUP Oxford; 2001.

24. Torrance GW. Measurement of health state utilities for economic appraisal. J Health Econ. 1986;5:1-30.

25. Oppe M, Devlin NJ, van Hout B, Krabbe PF, de Charro F. A program of methodological research to arrive at the new international EQ-5D-5L valuation protocol. Value Health. 2014;17:445-53.

26. Amin F, Mallick A, Lux A, O'Callaghan FJ. Quality of life in children and adults with tuberous sclerosis complex. Dev Med Child Neurol. 2016;58(Suppl. 1):68 (Abstract 125).

27. Amin S, Mallick AA, Lux A, O'Callaghan F. Quality of life in patients with Tuberous Sclerosis Complex (TSC). Eur J Paediatr Neurol. 2019;23:801-7.

28. Bennett B, Cooper A, Fladrowski C, Evanochko C, Neary M, Vieira $\mathbf{J}$, et al. Assessing quality of life (QOL) in patients with seizures associated with tuberous sclerosis complex (TSC). Epilepsia. 2018;59(Suppl. 3):S331-2 (Abstract p724).

29. Bennett B, Cooper A, Fladrowski C, Evanochko C, Neary MP, Vieira J, et al. Patient and caregiver web-based survey: health utilities in tuberous sclerosis complex (TSC) patient population. Value Health. 2018;21(Suppl. 3):S349 (Abstract PND123).

30. Fong CY, Ng K, Kong AN, Ong LC, Rithauddin MA, Thong MK, et al. Quality of life of children with tuberous sclerosis complex. Arch Child. 2019;104:972-8.

31. Kingswood JC, Crawford P, Johnson SR, Sampson JR, Shepherd $\mathrm{C}$, Demuth D, et al. The economic burden of tuberous sclerosis complex in the UK: a retrospective cohort study in the Clinical Practice Research Datalink. J Med Econ. 2016;19:1087-98.

32. Bernier A, Landry JS, Kristof AS, Carmant L, Major P. 2. Characterization of the tuberous sclerosis complex population in the province of Quebec: healthcare services utilization and long term outcome. Clin Neurophysiol. 2016;127:e160.

33. Demuth D, Nasuti P, Lucchese L, Gray L, Pinnegar A, Magestro M. Economic impact of patients with tuberous sclerosis complex (TSC) in the UK: a retrospective database analysis in the clinical practice research datalink (CPRD). Value Health. 2014;17:A137 (Abstract PHS69).

34. Kingswood JC, Nasuti P, Patel K, Myland M, Siva V, Gray E. The economic burden of tuberous sclerosis complex in UK patients with renal manifestations: a retrospective cohort study in the clinical practice research datalink (CPRD). J Med Econ. 2016;19:1116-26.

35. Lennert B, Farrelly E, Sacco P, Pira G, Frost M. Resource utilization in children with tuberous sclerosis complex and associated seizures: a retrospective chart review study. J Child Neurol. 2013;28:461-9.

36. Marques R, Belousova E, Benedik MP, Carter T, Cottin V, Curatolo $\mathrm{P}$, et al. Treatment patterns and use of resources in patients with tuberous sclerosis complex: insights from the TOSCA registry. Front Neurol. 2019;10:1144.

37. Skalicky AM, Rentz AM, Liu Z, Wheless JW, Pelletier CL, Dunn $\mathrm{DW}$, et al. The burden of subependymal giant cell astrocytomas associated with tuberous sclerosis complex: results of a patient and caregiver survey. J Child Neurol. 2015;30:563-9.

38. Dunn D, Rentz A, Skalicky A, Pelletier C, Liu Z, Prestifilippo J, et al. Characteristics and burden of subependymal giant cell astrocytomas (SEGAs) in patients with tuberous sclerosis complex: results of a patient and caregiver survey. Neurology. 2013;80(7 Suppl.):P07.016 (Abstract).

39. Jansen AC, Belousova E, Benedik MP, Carter T, Cottin V, Curatolo $\mathrm{P}$, et al. Quality of life and burden of disease in tuberous sclerosis complex (TSC): findings from TOSCA research project. J Intellect Disabil Res. 2017;61(9):829 (Abstract).

40. Bar C, Ghobeira R, Azzi R, Ville D, Riquet A, Touraine R, et al. Experience of follow-up, quality of life, and transition from 
pediatric to adult healthcare of patients with tuberous sclerosis complex. Epilepsy Behav. 2019;96:23-7.

41. Kopp CM, Muzykewicz DA, Staley BA, Thiele EA, Pulsifer MB. Behavior problems in children with tuberous sclerosis complex and parental stress. Epilepsy Behav. 2008;13:505-10.

42. Both P, Ten Holt L, Mous S, Patist J, Rietman A, Dieleman G, et al. Tuberous sclerosis complex: concerns and needs of patients and parents from the transitional period to adulthood. Epilepsy Behav. 2018;83:13-21.

43. Amin S, Lux A, Khan A, O'Callaghan F. Sirolimus ointment for facial angiofibromas in individuals with tuberous sclerosis complex. Int Sch Res Not. 2017;2017:8404378.

44. Crall C, Valle M, Kapur K, Dies KA, Liang MG, Sahin M, et al. Effect of angiofibromas on quality of life and access to care in tuberous sclerosis patients and their caregivers. Pediatr Dermatol. 2016;33:518-25.

45. Vergeer M, Ranitz-Greven WL, Neary MP, Ionescu-Ittu R, Emond B, Sheng Duh M, et al. Epilepsy, impaired functioning, and quality of life in patients with tuberous sclerosis complex. Epilepsia Open. 2019;4:581-92.

46. McDonald A, Goodwin J, Roberts S, Fish L, Vaughan B, Cooper A, et al. "We've made the best of it. But we do not have a normal life": families' experiences of tuberous sclerosis complex and seizure management. J Intellect Disabil Res. 2019;63:947-56.

47. Skalicky AM, Rentz AM, Liu Z, Said Q, Nakagawa JA, Frost $\mathrm{MD}$, et al. Economic burden, work, and school productivity in individuals with tuberous sclerosis and their families. J Med Econ. 2018;21:953-9.

48. Tritton T, Bennett B, Brohan E, Grant L, Cooper A, Fladrowski C, et al. Health utilities and quality of life in individuals with tuberous sclerosis complex (TSC) who experience epileptic seizures: a web-based survey. Epilepsy Behav. 2019;92:213-20.

49. Krueger DA, Care MM, Holland K, Agricola K, Tudor C, Mangeshkar P, et al. Everolimus for subependymal giant-cell astrocytomas in tuberous sclerosis. N Engl J Med. 2010;363:1801-11.

50. Liang S, Li A, Zhao M, Jiang H, Yu S, Meng X, et al. Epilepsy surgery in tuberous sclerosis complex: emphasis on surgical candidate and neuropsychology. Epilepsia. 2010;51:2316-21.
51. Liang S, Zhang J, Yang Z, Zhang S, Cui Z, Cui J, et al. Long-term outcomes of epilepsy surgery in tuberous sclerosis complex. J Neurol. 2017;264:1146-54.

52. Liu S, Yu T, Guan Y, Zhang K, Ding P, Chen L, et al. Resective epilepsy surgery in tuberous sclerosis complex: a nationwide multicentre retrospective study from China. Brain. 2020;143:570-81.

53. Roth J, Olasunkanmi A, MacAllister WS, Weil E, Uy CC, Devinsky $\mathrm{O}$, et al. Quality of life following epilepsy surgery for children with tuberous sclerosis complex. Epilepsy Behav. 2011;20:561-5.

54. Zamponi N, Petrelli C, Passamonti C, Moavero R, Curatolo P. Vagus nerve stimulation for refractory epilepsy in tuberous sclerosis. Pediatr Neurol. 2010;43:29-34.

55. Graffigna G, Bosio C, Cecchini I. Assisting a child with tuberous sclerosis complex (TSC): a qualitative deep analysis of parents' experience and caring needs. BMJ Open. 2013;3:e003707.

56. Toldo I, Brasson V, Miscioscia M, Bugin S, Manara R, Nosadini M, et al. Neuropsychiatric disorders and psychopathology of children and adolescents with tuberous sclerosis. Eur J Paediatr Neurol. 2017;21(Suppl. 1):e4 (Abstract OC7).

57. The Office for National Statistics (ONS). 2011 UK census [Internet]. 2011. https://www.ons.gov.uk/census. Accessed 25 Oct 2020.

58. CHTE methods review. NICE HRQL Task and Finish Group report. [Internet]. 2020. p. 1-115. https://www.nice.org.uk/Media/ Default/About/what-we-do/our-programmes/nice-guidance/chtemethods-consultation/Health-related-quality-of-life-task-and-finish-group-report.docx. Accessed 26 Nov 2020.

59. Rowen D, Brazier J, Wong R, Wailoo A. Measuring and valuing health-related quality of life when sufficient EQ-5D data is not available. NICE DSU report (page 48) [Internet]. 2020. http:// nicedsu.org.uk/measuring-and-valuing-health-related-qualityof-life-when-sufficient-data-is-not-directly-observed/. Accessed 2 Jun 2021.

60. Lipman SA. Time for tele-TTO? Lessons learned from digital interviewer-assisted time trade-off data collection. Patient. 2020. https://doi.org/10.1007/s40271-020-00490-z. 